\title{
Development of a dedicated repertoire and food frequency questionnaire for estimating dietary fiber intake taking into account prebiotic (oligo) saccharides
}

\author{
Audrey M. Neyrinck ${ }^{1}$, Julie-Anne Nazare,3, Monique Sothier $^{2,3}$, Laurie Vandenberghe ${ }^{2,3}$, \\ Camille Amadieu ${ }^{1}$, Julie Rodriguez ${ }^{1}$, Maud Alligier ${ }^{2,3}$, Martine Laville ${ }^{2,3}$ and \\ Nathalie M. Delzenne \\ ${ }^{1}$ Louvain Drug Research Institute, Université catholique de Louvain, Brussels, Belgium, \\ ${ }^{2}$ Rhône-Alpes Research Center for Human Nutrition, Lyon, France and \\ ${ }^{3}$ CarMeN Laboratory, Université Claude Bernard Lyon1, Hospices Civils de Lyon, CENS, FCRIN/FORCE Network, \\ Lyon, France
}

\begin{abstract}
Rationale: The scientific rationale for the recommendation in dietary fiber (DF) intake comes from the recognition of their health benefits. European Food Safety Authority (EFSA) recommend $25 \mathrm{~g} / \mathrm{d}$, based on the amount needed for intestinal laxation. Gut microbiota related health benefits are not yet included in the current dietary recommendations. Appropriate (and consensual) quantitative and qualitative analysis of DF are missing in food composition tables. One of the aim of the FiberTAG ${ }^{(1)}$ project was to develop a dedicated database and a food frequency questionnaire (FFQ) allowing DF intake estimation including prebiotic (oligo)saccharides.

Methods: A repertoire of DF detailing total, soluble and insoluble DF in food products consumed in Europe has been established based on the Souci-Fachmann-Kraut database, completed for soluble versus insoluble DF () and for prebiotic (oligo)saccharide levels using published data. The FiberTAG FFQ was submitted to healthy volunteers ( 7 men, 8 women; mean age: 21 yrs; mean BMI: $22 \mathrm{~kg} /$ $\mathrm{m} 2$ ) and DF intakes were calculated using the new FiberTAG repertoire.

Results: The FiberTAG repertoire detailed DF in 400 food items allocated into 4 categories (fruits, vegetables, cereal products and others) and 15 subcategories. The fiberTAG FFQ consists in 280 items regrouped in 4 high DF-containing food groups (vegetables including legumes, fruits including nuts and seed, cereal products and others) using frequency scale (6 categories), photographs for portion size and taking into account the seasonal occurrence for some vegetables or fruits. Data obtained from FiberTAG FFQ indicate a total DF intake of $36.44 \mathrm{~g} / \mathrm{d}$. This reveals a higher DF intake compared to previously reported intake for adults in Europe ranging from 16 to $24 \mathrm{~g} / \mathrm{d}$ based on $24 \mathrm{~h}$-recall or 3-7 days records ${ }^{(2)}$. This difference might be explained by a more adequate inclusion of fruits and vegetables intake in FFQ than with other methods, as previously reported ${ }^{(3)}$. Our data also showed that fructan, inulin and fructo-oligosaccharides intakes were 4.18, 2.95 and $1.00 \mathrm{~g} / \mathrm{day}$, respectively whereas galacto-oligosaccharides intake was $0.28 \mathrm{~g} / \mathrm{d}$.
\end{abstract}

Conclusion: The new FiberTAG DF repertoire and FFQ are tools to evaluate the total amount of DF including prebiotic (oligo)saccharides ingested by healthy volunteers. The FiberTAG project generates scientific knowledge that helps to take into account microbiota-nutrient interactions to establish DF intake recommendations as part of healthy lifestyles.

\section{Conflict of Interest}

There is no conflict of interest

\section{Reference}

1. FiberTAG project https://www fibertag ew/

2. Stephen (2017) Nutr Res Rev 30, 149-190.

3. Riordan (2016) Public Health Nutrition 20, 417-448. 